\title{
Phase separation in a bidirectional two-lane asymmetric exclusion process
}

\author{
Rui Jiang ${ }^{1,2}$, Katsuhiro Nishinari ${ }^{2}$, Mao-Bin $\mathrm{Hu}^{1}$, Yong-Hong $\mathrm{Wu}^{3}$, Qing-Song $\mathrm{Wu}^{1}$ \\ ${ }^{1}$ School of Engineering Science, University of Science and technology of China, Hefei 230026, China \\ 2 Department of Aeronautics and Astronautics, School of Engineering, \\ University of Tokyo, Hongo, Bunkyo-ku, Tokyo 113-8656, Japan and \\ 3 Department of Mathematics and Statistics, Curtin University of Technology, Perth WA6845, Australia
}

\begin{abstract}
This paper studies a bidirectional two-lane asymmetric exclusion process, in which particles move in opposite direction on the two lanes. Interaction between the two lanes is implemented as follows: particle hops with rate $p$ when there is a particle at the same site in the other lane, otherwise it hops with rate 1 . It is shown that under periodic boundary conditions, a plateau will form on the fundamental diagram if $p<1$. This plateau corresponds to a phase separation phenomenon. We have compared the phase separation with those reported in previous works, and it is shown that the mechanism of phase separation in our model is different from previous ones. A possible phase separation mechanism is proposed, i.e., the system always tries to maximize the probability that particles could hop with rate 1. A simple mean field approximation and a 2-cluster mean field approach have been applied to calculate the steady current. It is shown that the results of the 2-cluster mean field approach are much closer to the simulations.
\end{abstract}

PACS numbers: 02.50.Ey, 05.70.Ln, 05.70.Fh, 82.20.Mj

\section{INTRODUCTION}

Driven diffusive systems out of equilibrium have attracted much attention due to their rich and complex dynamic phase behaviors. An important class of such models is the asymmetric exclusion process (ASEP) describing particles hopping with hard-core repulsion along a 1D lattice [1]. Such systems provide a good description of traffic flow [2], the kinetics of biopolymerization [3], polymer dynamics in dense media [4], diffusion through membrane channels [5], dynamics of motor proteins moving along rigid filaments [6], etc. Despite their simplicity, the ASEP and related models show a range of nontrivial macroscopic phenomena, such as boundary induced phase transitions [7], spontaneous symmetry breaking [8], shock fronts [9].

Phase separation is another nontrivial phenomenon observed in ASEP. In 1998, Arndt, Heinzel and Rittenberg (AHR) [10] reported two kinds of phase separation in their three-species model, the fully separated state and the mixed state. In the former state, the three species are well separated from each other. Similar phenomenon is also reported by Evans et al. [11] in a related ABC model. In the latter state, spatial condensation occurs and a macroscopic cluster of particles will coexist with uniform low density region. Nevertheless, through an analytical analysis, Rajewsky et al. proved that the mixed state in AHR model is a mere finite-size effect and should vanish in sufficiently large system $\left(\mathrm{O}\left(10^{70}\right)\right)[12]$.

The research group of Zia and Schmittmann has reported phase separation, which is named coarsening phenomenon in their papers, in 2D exclusion process [13]. They also found that the phase separation phenomenon could be observed in a two-lane exclusion process [14]. Kafri et al. have proposed a general criterion for the existence of phase separation and pointed out that the phase separation in the two-lane exclusion process also vanishes in extremely large system [15]. Moreover, they introduced a class of models which is shown to exhibit true phase separation [16]. Nevertheless, Georgiev et al. indicated that it is still interesting to investigate the evolution to phase separation in finite systems because the phase separation does not vanish until the system sizes reach $\mathrm{O}\left(10^{70}\right)$, which is irrelevant for all conceivable earthbound systems (e.g., pedestrian traffic and biological motors) [17]. Recently, Schütz have discussed the criterion of Kafri et al. and pointed out some assumptions which require rigorous proof [18].

In this paper, we report phase separation in a bidirectional two-lane ASEP, which is different from those mentioned above. In our model, particles move in opposite direction on the two lanes and a particle reduces its speed when there is another particle at the same site in the other lane. It is shown that a plateau will form on the fundamental diagram, which corresponds to the phase separation phenomenon. A possible phase separation mechanism is proposed. A simple mean field approximation and a 2-cluster mean field approach have been applied to calculate the steady current.

The paper is organized as follows. The model is introduced in section II. The Monte Carlo simulation results are discussed in section III. In section IV, we compare the phase separation with those reported in previous works in details. In section V, mean field analysis is presented. Conclusions are given in section VI. 


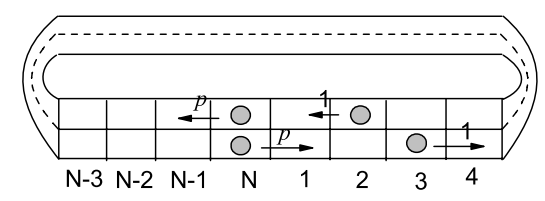

FIG. 1: Sketch of the model. The symbols above the arrows show the hopping rate.

\section{MODEL}

In our model, the inter-lane interaction is implemented as particles slow down when there is a particle at the same site in the other lane. This is slightly different from the model presented by Lee et al. [19], in which particles slow down in the vicinity of approaching particles in the other lane. The difference does not bring out qualitative change of results. This is easy to understand. Assume initial configuration of our model is $\left(\tau_{1}, \tau_{2}, \ldots, \tau_{N}\right)$ and $\left(\sigma_{1}, \sigma_{2}, \ldots, \sigma_{N}\right)$ (see next paragraph for definition of $\tau$ and $\sigma$ ), if we set initial configuration of the model in [19] as $\left(\tau_{1}, \tau_{2}, \ldots, \tau_{N}\right)$ and $\left(\sigma_{N}, \sigma_{1}, \ldots, \sigma_{N-1}\right)$ (i.e., shift particles on lane 2 to their right neighbor sites), then these two models will exhibit exactly same results.

The details of our model are as follows. Consider two parallel one-dimensional lanes, each with $N$ sites (see Fig.1). Particles move to the right on lane 1 and move to the left on lane 2. The state of the system is characterized by two sets of occupation numbers $\left(\tau_{1}, \tau_{2}, \ldots, \tau_{N}\right)$ and $\left(\sigma_{1}, \sigma_{2}, \ldots, \sigma_{N}\right)$ for the first and second lanes. If site $i$ of lane 1 is occupied by a particle, $\tau_{i}=1$, and zero otherwise. Similarly $\sigma_{i}=1$ if site $i$ of lane 2 is occupied by a particle, and $\sigma_{i}=0$ if the site is empty. In an infinitesimal time interval $d t$, a particle at site $i$ hops to its right (left) empty site on lane 1 (2) with the probability $d t$ if there is no particle at the same site on the other lane, and with the reduced probability pdt otherwise. Namely,

$$
\begin{gathered}
\left(\tau_{i}, \tau_{i+1}\right)=(1,0) \rightarrow(0,1) \text { with rate }\left\{\begin{array}{ll}
1 & \text { if } \sigma_{i}=0 \\
p & \text { if } \sigma_{i}=1
\end{array} \text { for } 1 \leq i<N\right. \\
\left(\sigma_{i}, \sigma_{i+1}\right)=(0,1) \rightarrow(1,0) \text { with rate }\left\{\begin{array}{ll}
1 & \text { if } \tau_{i+1}=0 \\
p & \text { if } \tau_{i+1}=1
\end{array} \text { for } 1 \leq i<N\right.
\end{gathered}
$$

For periodic boundary conditions, we have (see Fig.1)

$$
\begin{gathered}
\left(\tau_{N}, \tau_{1}\right)=(1,0) \rightarrow(0,1) \text { with rate } \begin{cases}1 & \text { if } \sigma_{N}=0 \\
p & \text { if } \sigma_{N}=1\end{cases} \\
\left(\sigma_{N}, \sigma_{1}\right)=(0,1) \rightarrow(1,0) \text { with rate } \begin{cases}1 & \text { if } \tau_{1}=0 \\
p & \text { if } \tau_{1}=1\end{cases}
\end{gathered}
$$

In this paper, the random sequential update is adopted. We would like to mention that our model is a special case of the models proposed in Ref.[20]. By setting

$$
\alpha=\varepsilon=1, \beta=\gamma=p, \alpha_{L}=\beta_{L}=\gamma_{L}=\varepsilon_{L}=0,
$$

the antisymmetric model in Ref.[20] reduces to our model. However, Eq.(5) (except in the case of $p=1$, which is trivial) does not meet the solvable condition, see Eq.(2) in Ref.[20]. Thus, phase separation is not revealed in Ref.[20].

\section{SIMULATION RESULTS}

In this section, the Monte Carlo simulation results are presented. In the simulations, a transient time of $2 \times 10^{9}$ time steps is discarded. We gather data for $18 \times 10^{9}$ time steps. The system size $N=10000$ is used unless otherwise 


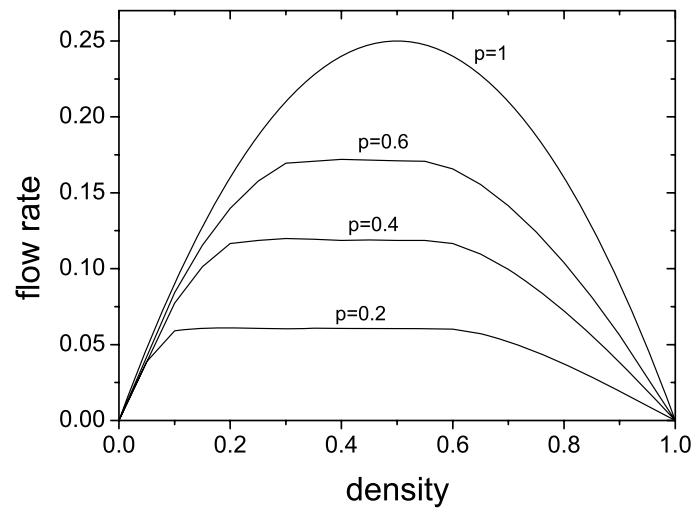

FIG. 2: Fundamental diagram of the bidirectional two-lane system.

mentioned. In this paper, we only study the case $\rho_{1}=\rho_{2}=\rho$, and the situation arising from $\rho_{1} \neq \rho_{2}$ will be reported elsewhere. Here $\rho_{1}$ and $\rho_{2}$ denote density on lanes 1 and 2 , respectively.

Fig.2 shows the fundamental diagram of the system. One can see that when $p<1$, two critical densities $\rho_{c 1}$ and $\rho_{c 2}$ appear. When $\rho<\rho_{c 1}$, the flow rate increases with the increase of $\rho$; when $\rho>\rho_{c 2}$, the flow rate decreases with the increase of $\rho$. In the intermediate density range $\rho_{c 1}<\rho<\rho_{c 2}$, a plateau forms. Fig.3 shows typical spatiotemporal patterns at small density $\left(\rho<\rho_{c 1}\right)$, high density $\left(\rho>\rho_{c 2}\right)$, and intermediate density $\left(\rho_{c 1}<\rho<\rho_{c 2}\right)$, respectively. It can be seen that the traffic flow is homogeneous at small density and high density. Nevertheless, a phase separation is observed at intermediate density. The system separates into high density region and low density region. The density of the high density region equals to $\rho_{c 2}$ and the density of the low density region equals to $\rho_{c 1}$. The high density region performs a random walk in the system. With the increase (decrease) of density in the plateau region, the flow rate does not change, but the high (low) density region gradually expands until it finally occupies the whole system. Then the phase separation transits into homogeneous high (low) density phase. Furthermore, it can also be seen that when phase separation appears, the synchronization of densities on two lanes could be observed.

Next we provide a possible explanation of the phase separation. To this end, we investigate the situation in a single vertical cluster. We define $P_{0}$ as a probability to find a vertical cluster with both lattice sites empty, $P_{1}$ and $P_{2}$ as probabilities to have a half-empty vertical cluster with a particle at lane 2 or 1 , respectively, and $P_{3}$ as a probability to have particles at both lattice sites (see Fig.4). Fig.5 shows the dependence of four probabilities on the density $\rho$. At the same time, the flow rate is also shown. One can see that $P_{0}$ decreases and $P_{3}$ increases with the increase of density.

Now we focus on $P_{1}$ (note that $P_{2}=P_{1}$ ). It increases with the decrease of density from $\rho=1$. When $\rho=\rho_{c 2}$, the maximum value of $P_{1}$ is reached. Then the system transits into phase separation, in which the maximum value of $P_{1}$ is maintained in high density region. On the other hand, a smaller value of $P_{1}$ exists in low density region. As a result, the averaged value of $P_{1}$ decreases linearly with the decrease of $\rho$. When $\rho<\rho_{c 1}$, the system becomes homogeneous again and $P_{1}$ continues to decrease with the decrease of $\rho$.

Based on this result, we argue that the phase separation mechanism is that the system always tries to maximize $P_{1}$ (i.e., the probability that particles could hop with rate 1) in the whole system and if not possible, in part of the system. Specifically, when $P_{1}$ reaches the maximum at $\rho=\rho_{c 2}$, it will decrease with the decrease of $\rho$ if phase separation does not occur (see Fig.6(b)). Nevertheless, the system self-organizes into phase separation to maintain the maximum $P_{1}$ in part of system (i.e., in the high density region). In section $\mathrm{V}$, this mechanism is adopted to calculate the flow rate corresponding to the plateau.

Finally we check whether the phase separation is due to finite size effect. To this end, we employ the criterion proposed by Kafri et al. [15]. We assume that the current of a finite domain of size $n$ takes the form $J_{n}=J_{\infty}(1+b / n)$. Fig.7 shows the plot of $\Delta_{n}=J_{n} / J_{\infty}-1$ versus $n$. This is fulfilled by considering an open two-lane system of length $n$ with particles injected with rate 1 and ejected with rate 1 or $p$ at the boundaries. It can be seen that in this case $b \approx 4>2$. This demonstrates that it is a true phase separation. 


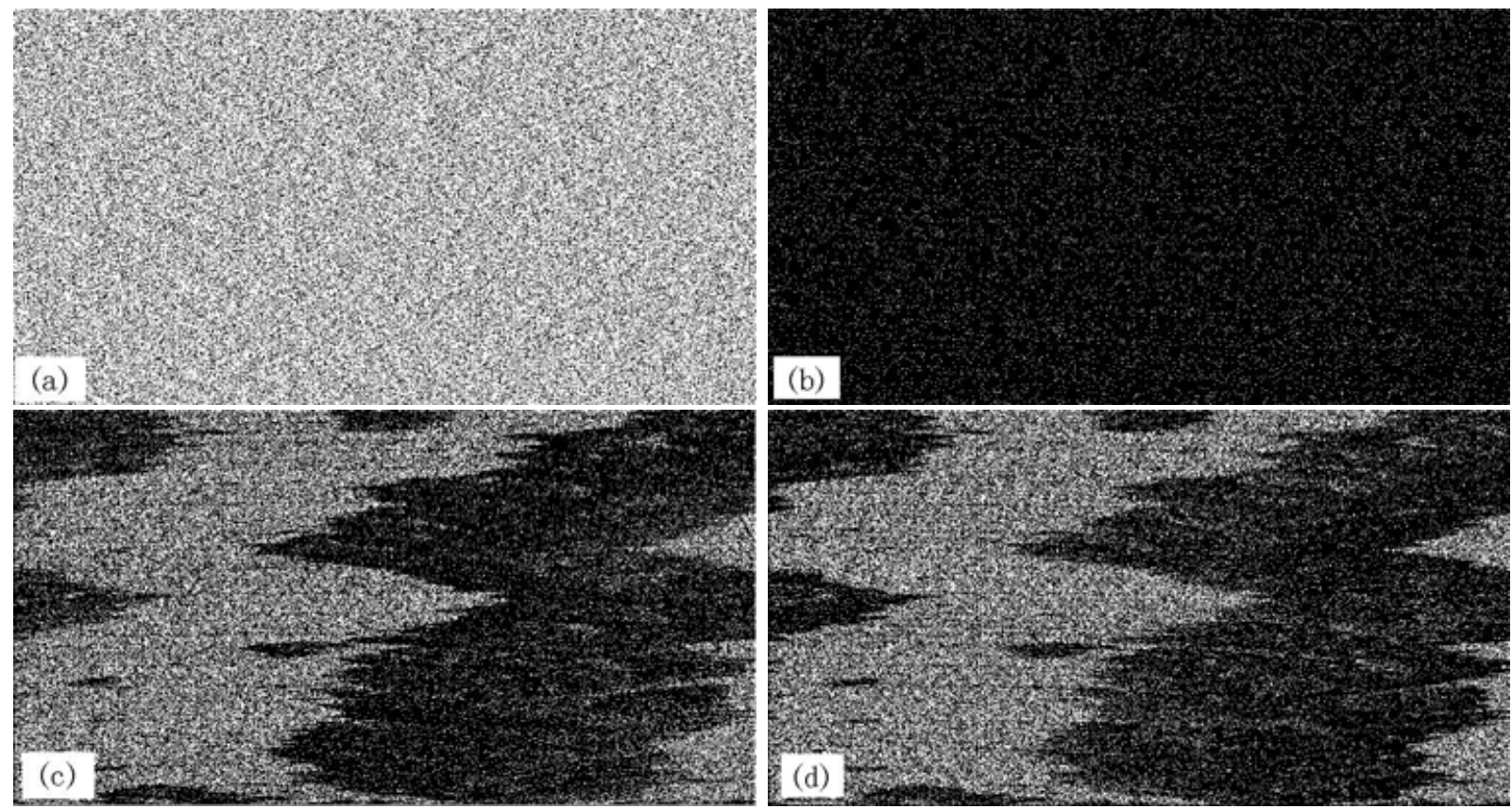

FIG. 3: Typical spatiotemporal patterns at (a) small density $(\rho=0.1)$; (b) high density $(\rho=0.7)$, and (c,d) intermediate density $(\rho=0.4)$. (c) shows traffic flow on lane 1 and (d) shows traffic flow on lane 2 . Initially the particles are distributed randomly on the two lanes. 1000 snapshots of the system are shown every $3 \times 10^{5}$ time steps. The slow down probability $p=0.4$, and the system size is $N=1000$. The time is increasing in upward direction.

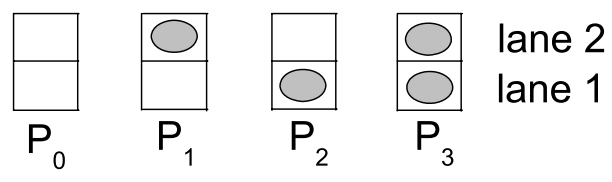

FIG. 4: Four possible states of a vertical cluster.
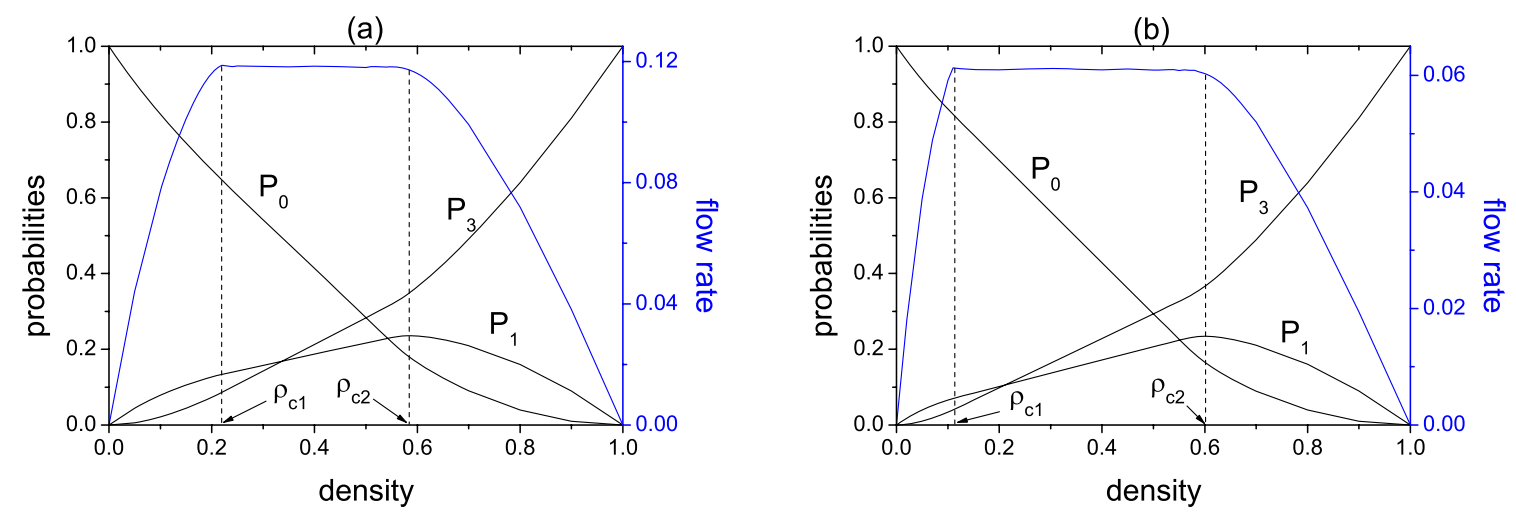

FIG. 5: (color online) The dependence of the four probabilities and the flow rate on the density. (a) $p=0.4$; (b) $p=0.2$. Note that $P_{1}=P_{2}$ and $\rho=P_{1}+P_{3}$ (see Eq.(6)). 


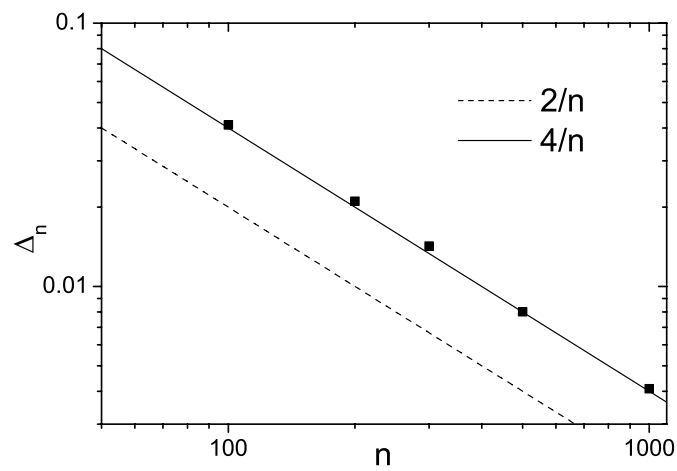

FIG. 6: The finite-size corrections to the current $\Delta_{n}$ in the two-lane model with open boundary conditions, for different system size $n$. Here $J_{\infty} \approx 0.06115$ from numerical simulation. The parameter $p=0.2$.

\section{DISCUSSION}

In this section, we compare the phase separation in our model with those reported in previous works in details.

Firstly, we compare the phase separation in this paper with that in two-lane two-species model [14] and the mixed state in AHR model [10]. The phase separation will disappear when the density exceeds $\rho_{c 2}$ in our model while it persists until $\rho=1$ in those two models. The is because in those two models, clusters will finally coarse into a compact jam in which there is essentially no vacancy existing between particles. In contrast, the high density region does not coarse into compact jam in our model. More importantly, the phase separation is due to finite size effect in those two models while it is true phase separation in our model.

Phase separation is also observed in systems with defect sites or particles [19,21-26], in which the high density region either remains stationary (in case of defect sites) or moves constantly with the defect (in case of defect particles) [36]. In contrast, in our model, instead of remaining stationary or moving constantly, the high density region in phase separation state performs a random walk.

The models with slow-to-start (s2s) rule (see, e.g.Ref.[27]), the bus route (BR) model [28] and the ant-trail (AT) model $[29,30]$ also exhibit phase separation. In the s2s model, the high density region moves upstream with constant velocity depending on the randomization $p_{0}$ at velocity $v=0$ (see Fig.6 in Ref.[27]). In the BR model and the AT model, the high density region [37] moves downstream with constant velocity depending on the gap of the leading particle (see, e.g., Fig.1 in Ref.[30]). Different from our model, the phase separation could be observed when the global density is high (low) until $\rho=1(\rho=0)$ in s2s (BR and AT) model and the flow rate decreases (increases) with the increase of density in phase separation state.

Finally, an interesting phase separation is reported in a two-lane model under open boundary condition (which is another special case of the models in Ref.[20]), see Fig.4 in Ref.[31]. This is due to a give value of current can be realized in different ways, as shown in Fig.2 in Ref.[31]. However, since the stationary state has a product form, the current can be obtained exactly and accordingly the phase separation disappears under periodic boundary condition.

To summarize, the phase separation observed in our model is different from those reported before. Presently we could only provide a possible explanation of its mechanism, and further investigations are needed to explore the exact origin of the phase separation.

\section{MEAN FIELD ANALYSIS}

In this section, a simple mean field theory and a 2-cluster mean field theory are used to calculate the flow rate. Note that the mean field theory assumes that the traffic flow is always homogeneous at all densities. Therefore, the flow rate corresponding to the plateau, $J_{\max }$, could not be directly predicted from mean field theoy. Nevertheless, the critical density $\rho_{c 2}$ could be obtained by identifying the maximum value of $P_{1}$ as described in previous section. Then $J_{\max }$ and the other critical density $\rho_{c 1}$ could be obtained accordingly. 


\section{A. Simple mean field theory}

In simple mean field theory, we study the four probabilities $P_{0}-P_{3}$. The occupation of vertical clusters is assumed to be independent of the position along the channels. The conservation of probability requires that

$$
P_{0}+P_{1}+P_{2}+P_{3}=1
$$

Furthermore, the definition of density gives

$$
\begin{aligned}
& P_{2}+P_{3}=\rho . \\
& P_{1}+P_{3}=\rho .
\end{aligned}
$$

The evolution of four probabilities can be described via Master equations. Specifically, for $P_{0}, P_{1}, P_{2}, P_{3}$, we have

$$
\begin{aligned}
& \frac{d P_{0}}{d t}=\frac{d P_{3}}{d t}=-2 P_{0} P_{3} p+2 P_{1} P_{2} . \\
& \frac{d P_{1}}{d t}=\frac{d P_{2}}{d t}=-2 P_{1} P_{2}+2 P_{0} P_{3} p .
\end{aligned}
$$

In stationary state, we have $\frac{d P_{0}}{d t}=\frac{d P_{1}}{d t}=\frac{d P_{2}}{d t}=\frac{d P_{3}}{d t}=0$. Thus,

$$
P_{0} P_{3} p=P_{1} P_{2} \text {. }
$$

Substituting Eqs.(6-8) into (11), we have

$$
(p-1) P_{3}^{2}+[2 \rho(1-p)+p] P_{3}-\rho^{2}=0 .
$$

The solutions are

$$
P_{3}=\frac{2 \rho(p-1)-p \pm \sqrt{[2 \rho(p-1)-p]^{2}+4 \rho^{2}(p-1)}}{2(p-1)}
$$

The solution related to "-" needs to be discarded because only the solution related to "+" leads to $P_{3}=\rho^{2}$ when $p \rightarrow 1$. Substituting

$$
P_{3}=\frac{2 \rho(p-1)-p+\sqrt{[2 \rho(p-1)-p]^{2}+4 \rho^{2}(p-1)}}{2(p-1)}
$$

into Eqs.(6-8), $P_{0}, P_{1}, P_{2}$ can be obtained. Consequently, the flow rates on lanes 1 and 2 are

$$
\begin{aligned}
& J_{1}=P_{2} P_{0}+P_{2} P_{1}+P_{3} P_{0} p+P_{3} P_{1} p . \\
& J_{2}=P_{1} P_{0}+P_{1} P_{2}+P_{3} P_{0} p+P_{3} P_{2} p .
\end{aligned}
$$

The mean field results of $J=J_{1}=J_{2}$ are shown in Fig.7(a). Fig.7(b) shows the plot of $P=P_{1}=P_{2}$ versus $\rho$. One can see that the maximum value of $P_{1}$ is achieved at $\rho=0.5$. This implies that $\rho_{c 2}=0.5$. Having $\rho_{c 2}, J_{\text {max }}$ and $\rho_{c 1}$ could be easily determined. The result is compared with simulation results in Fig.8. One can see that there is large deviation between the analytical results and the simulation results. This is because the correlation between clusters is neglected in simple mean field theory.

\section{B. 2-cluster mean field theory}

To consider the correlation between clusters, we use the cluster mean field theory. We define a $n$-cluster to be a collection of $n$ successive vertical clusters and denote the probability of finding an $n$-cluster in the state $\left(\sigma_{1}, \sigma_{2}, \ldots, \sigma_{n}\right)$ in the stationary state of the system by the symbol $P_{n}\left(\sigma_{1}, \sigma_{2}, \ldots, \sigma_{n}\right)$. In the general $n$-cluster approximation, one divides the lattice into "clusters" of length $n$ such that two neighboring clusters have $n-1$ vertical clusters in common 
(a)

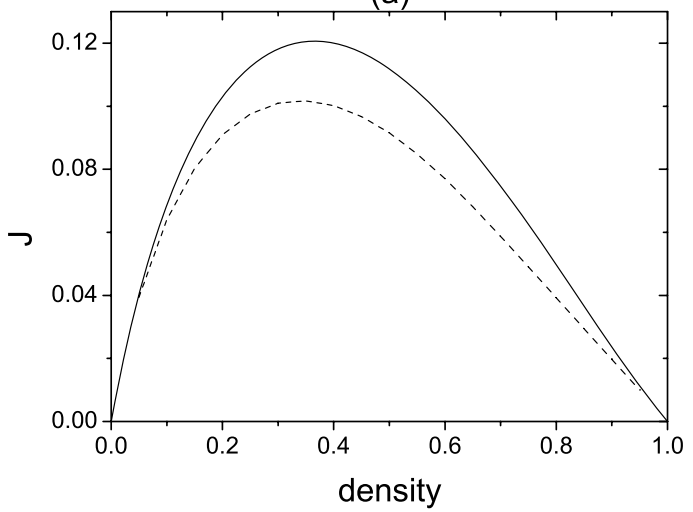

(b)

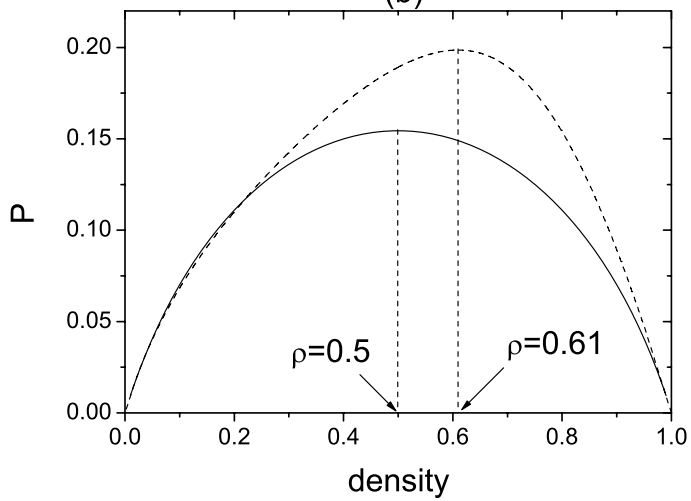

FIG. 7: (a) $J$ and (b) $P$ versus density. The solid lines are from simple mean field theory, the dashed lines are from 2-cluster mean field theory. The parameter $p=0.2$.

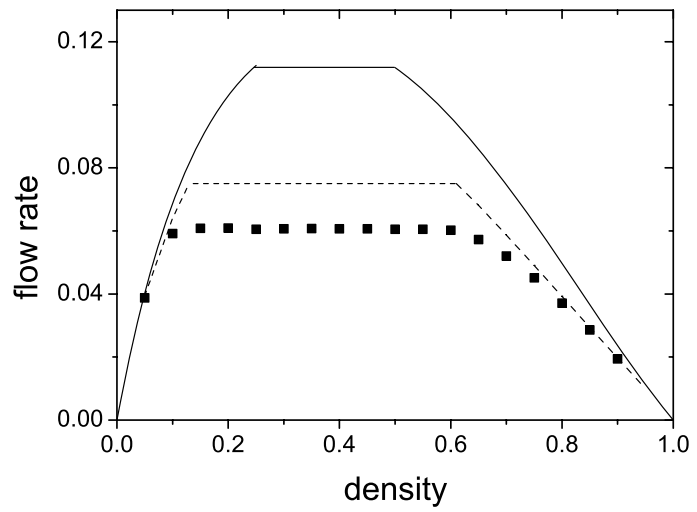

FIG. 8: Flow rate versus density. The solid lines are from simple mean field theory, the dashed lines are from 2-cluster mean field theory, the scattered data are from simulations. The parameter $p=0.2$.

[2,32-35]. Since this mean field method becomes increasingly unwieldy as the size of the cluster increases, we only show results for the 2-cluster mean field theory. In this case, the state of the 2-cluster $\sigma_{i}, \sigma_{i+1}$ at time $t+1$ depends on the state of the 4 -cluster $\left(\sigma_{i-1}, \sigma_{i}, \sigma_{i+1}, \sigma_{i+2}\right)$ at time $t$. As a result, the master equation for the two site probability $P(3,3)$ is

$$
\begin{aligned}
\frac{d P(3,3)}{d t}= & -P(2,3,3,0) p-P(2,3,3,1) p-P(2,3,3,2) p-P(2,3,3,3) p \\
& -P(0,3,3,0) p-P(0,3,3,1) p-P(0,3,3,2) p-P(0,3,3,3) p \\
& -P(0,3,3,1) p-P(1,3,3,1) p-P(2,3,3,1) p-P(3,3,3,1) p \\
& -P(0,3,3,0) p-P(1,3,3,0) p-P(2,3,3,0) p-P(3,3,3,0) p \\
& +P(0,3,2,1)+P(1,3,2,1)+P(2,3,2,1)+P(3,3,2,1) \\
& +P(0,3,2,3) p+P(1,3,2,3) p+P(2,3,2,3) p+P(3,3,2,3) p \\
& +P(2,1,3,0)+P(2,1,3,1)+P(2,1,3,2)+P(2,1,3,3) \\
& +P(3,1,3,0) p+P(3,1,3,1) p+P(3,1,3,2) p+P(3,1,3,3) p .
\end{aligned}
$$

Since 2-cluster approximation can be expressed mathematically as

$$
P\left(\sigma_{i-1}, \sigma_{i}, \sigma_{i+1}, \sigma_{i+2}\right)=P\left(\sigma_{i-1} \mid \underline{\sigma_{i}}\right) P\left(\sigma_{i}, \sigma_{i+1}\right) P\left(\underline{\sigma_{i+1}} \mid \sigma_{i+2}\right),
$$


with

$$
P\left(\sigma_{i-1} \mid \underline{\sigma_{i}}\right)=\frac{P\left(\sigma_{i-1}, \sigma_{i}\right)}{\sum_{\sigma_{i-1}} P\left(\sigma_{i-1}, \sigma_{i}\right)},
$$

Eq.(17) is simplified as

$$
\begin{aligned}
\frac{d P(3,3)}{d t}= & -\frac{P(2,3) P(3,3) p+P(0,3) P(3,3) p}{P(0,3)+P(1,3)+P(2,3)+P(3,3)}-\frac{P(3,3) P(3,1) p+P(3,3) P(3,0) p}{P(3,0)+P(3,1)+P(3,2)+P(3,3)} \\
& +\frac{P(3,2) P(2,1)+P(3,2) P(2,3) p}{P(2,0)+P(2,1)+P(2,2)+P(2,3)}+\frac{P(2,1) P(1,3)+P(3,1) P(1,3) p}{P(0,1)+P(1,1)+P(2,1)+P(3,1)} .
\end{aligned}
$$

In stationary state, we have $\frac{d P(3,3)}{d t}=0$. Thus,

$$
\begin{aligned}
& \frac{P(2,3) P(3,3) p+P(0,3) P(3,3) p}{P(0,3)+P(1,3)+P(2,3)+P(3,3)}+\frac{P(3,3) P(3,1) p+P(3,3) P(3,0) p}{P(3,0)+P(3,1)+P(3,2)+P(3,3)} \\
& \frac{P(3,2) P(2,1)+P(3,2) P(2,3) p}{P(2,0)+P(2,1)+P(2,2)+P(2,3)}+\frac{P(2,1) P(1,3)+P(3,1) P(1,3) p}{P(0,1)+P(1,1)+P(2,1)+P(3,1)} .
\end{aligned}=
$$

Similarly, we can obtain 15 other equations as shown in Appendix. Furthermore, the conservation of probability requires that

$$
\sum_{i=0,3}^{j=0,3} P(i, j)=1 .
$$

Furthermore, the definition of density gives

$$
\begin{aligned}
& P(3,3)+P(3,2)+P(3,1)+P(3,0)+P(1,3)+P(1,2)+P(1,1)+P(1,0)=\rho, \\
& P(3,3)+P(2,3)+P(1,3)+P(0,3)+P(3,1)+P(2,1)+P(1,1)+P(0,1)=\rho, \\
& P(3,3)+P(3,2)+P(3,1)+P(3,0)+P(2,3)+P(2,2)+P(2,1)+P(2,0)=\rho, \\
& P(3,3)+P(2,3)+P(1,3)+P(0,3)+P(3,2)+P(2,2)+P(1,2)+P(0,2)=\rho .
\end{aligned}
$$

Finally, the symmetry implies

$$
\begin{aligned}
& P(3,3)+P(3,2)+P(3,1)+P(3,0)=P(3,3)+P(2,3)+P(1,3)+P(0,3), \\
& P(2,3)+P(2,2)+P(2,1)+P(2,0)=P(3,2)+P(2,2)+P(1,2)+P(0,2), \\
& P(1,3)+P(1,2)+P(1,1)+P(1,0)=P(3,1)+P(2,1)+P(1,1)+P(0,1), \\
& P(3,0)+P(2,0)+P(1,0)+P(0,0)=P(0,3)+P(0,2)+P(0,1)+P(0,0) .
\end{aligned}
$$

To summarize, presently we have 16 parameters and 25 equations. Nevertheless, there are only 16 independent equations. For instance, we find that Eqs.(21, A1-A3, A5-A9, A11, A15, 22,23, 25-27) are one set of independent equations. We would like to mention that there are more than one set of independent equations. For example, Eqs.(21, A4, A6-A10, A12-A15, 22,23, 25, 26, 30) are another set of independent equations. Although analytical solutions of the equations could not be derived, numerical solutions could be obtained by the Newton iteration method.

Having the numerical solutions of the 16 probabilities $P(3,3), \ldots, P(0,0)$, the flow rates on lanes 1 and 2 could be expressed as

$$
\begin{aligned}
& J_{1}=p P(3,0)+p P(3,1)+P(2,0)+P(2,1) . \\
& J_{2}=p P(0,3)+p P(2,3)+P(2,1)+P(0,1) .
\end{aligned}
$$

The 2-cluster mean field results of $J=J_{1}=J_{2}$ and $P=P_{1}=P_{2}$ are also shown in Fig.7 [note that $P_{1}=$ $P(1,3)+P(1,2)+P(1,1)+P(1,0)$ and $\left.P_{2}=P(2,3)+P(2,2)+P(2,1)+P(2,0)\right]$. One can see that the maximum value of $P_{1}$ is achieved at $\rho=0.61$, which implies that $\rho_{c 2}=0.61$. Then $J_{\max }$ and $\rho_{c 1}$ can be determined. The 2-cluster mean field result is also compared with simulation results in Fig.8. One can see that the result is much closer to simulations. We believe if $n$-cluster $(n>2)$ mean field analysis is adopted, better agreement could be achieved. 


\section{CONCLUSIONS}

This paper has studied the two-lane two-way traffic flow under periodic boundary conditions. Particles move in opposite direction on the two lanes. The interaction between two lanes is implemented as particle hops with rate $p$ when there is a particle at the same site in the other lane.

It is found that a plateau will form on the fundamental diagram when $p<1$. The phase separation is observed corresponding to the plateau, while homogeneous traffic is observed beyond the plateau. It is verified that the phase separation is not a finite size effect, based on the criterion proposed by Kafri et al. [15].

We have compared the phase separation with those reported in previous works, and it is shown that the mechanism of phase separation in our model is different from previous ones. A possible explanation of the phase separation mechanism is proposed, i.e., the system always tries to maximize the probability that particles could hop with rate 1 in the whole system and if not possible, in part of the system. Exact origin of the phase separation still needs further investigations.

We have applied a simple mean field approximation and a 2-cluster mean field approach to calculate the steady current. The critical density $\rho_{c 2}$ is identified by locating the maximum value of $P_{1}$ and $P_{2}$. Accordingly, the flow rate $J_{\max }$ and the other critical density $\rho_{c 1}$ can be determined. It is shown that the results of the 2-cluster mean field approach are much closer to the simulation results than those from the simple mean field approximation.

We would like to mention that due to the different phase separation mechanism, the analytical method used in Ref.[29] (i.e., loose cluster approximation and zero range process mapping) could not be used in our model. We need to find a better analytical method for our model in the future work. This might be a tough task, similar to situation arising from ASEP with a defect site (presently there is still no better analytical method than mean field theory, see, e.g. Ref.[25]).

\section{APPENDIX A}

In this Appendix, we present the other 15 equations. From $\frac{d P(3,2)}{d t}=0$, we have

$$
\begin{aligned}
& \frac{P(2,3) P(3,2) p+P(0,3) P(3,2) p}{P(0,3)+P(1,3)+P(2,3)+P(3,3)}+\frac{P(3,2) P(2,1)+P(3,2) P(2,0)}{P(2,0)+P(2,1)+P(2,2)+P(2,3)}+\frac{P(3,2) P(2,1)+P(3,2) P(2,3) p}{P(2,0)+P(2,1)+P(2,2)+P(2,3)}= \\
& \frac{P(2,1) P(1,2)+P(3,1) P(1,2) p}{P(3,1)+P(2,1)+P(1,1)+P(0,1)}+P(2,3) p .
\end{aligned}
$$

From $\frac{d P(3,1)}{d t}=0$, we have

$$
\begin{aligned}
& \frac{P(0,3) P(3,1) p+P(2,3) P(3,1) p}{P(0,3)+P(1,3)+P(2,3)+P(3,3)}+P(3,1) p= \\
& \frac{P(2,1) P(1,1)+P(3,1) P(1,1) p}{P(0,1)+P(1,1)+P(2,1)+P(3,1)}+\frac{P(3,0) P(0,1)+P(3,0) P(0,3) p}{P(0,0)+P(0,1)+P(0,2)+P(0,3)}+\frac{P(3,3) P(3,1) p+P(3,3) P(3,0) p}{P(3,0)+P(3,1)+P(3,2)+P(3,3)} .
\end{aligned}
$$

From $\frac{d P(3,0)}{d t}=0$, we have

$$
\begin{aligned}
& \frac{P(0,3) P(3,0) p+P(2,3) P(3,0) p}{P(0,3)+P(1,3)+P(2,3)+P(3,3)}+\frac{P(3,0) P(0,1)+P(3,0) P(0,3) p}{P(0,3)+P(0,2)+P(0,1)+P(0,0)}+P(3,0) p= \\
& \frac{P(2,1) P(1,0)+P(3,1) P(1,0) p}{P(0,1)+P(1,1)+P(2,1)+P(3,1)}+\frac{P(3,2) P(2,0)+P(3,2) P(2,1)}{P(2,0)+P(2,1)+P(2,2)+P(2,3)}+P(2,1) .
\end{aligned}
$$

From $\frac{d P(2,3)}{d t}=0$, we have

$$
\begin{aligned}
& \frac{P(2,3) P(3,0) p+P(2,3) P(3,1) p}{P(3,0)+P(3,1)+P(3,2)+P(3,3)}+P(2,3) p= \\
& \frac{P(0,3) P(3,3) p+P(2,3) P(3,3) p}{P(0,3)+P(1,3)+P(2,3)+P(3,3)}+\frac{P(2,2) P(2,1)+P(2,2) P(2,3) p}{P(2,0)+P(2,1)+P(2,2)+P(2,3)}+\frac{P(2,0) P(0,3)+P(3,0) P(0,3) p}{P(0,0)+P(1,0)+P(2,0)+P(3,0)} .
\end{aligned}
$$

From $\frac{d P(2,2)}{d t}=0$, we have

$$
\begin{aligned}
& \frac{P(2,2) P(2,0)+P(2,2) P(2,1)}{P(2,0)+P(2,1)+P(2,2)+P(2,3)}+\frac{P(2,2) P(2,1)+P(2,2) P(2,3) p}{P(2,0)+P(2,1)+P(2,2)+P(2,3)} \\
& \frac{P(2,0) P(0,2)+P(3,0) P(0,2) p}{P(0,0)+P(1,0)+P(2,0)+P(3,0)}+\frac{P(0,3) P(3,2) p+P(2,3) P(3,2) p}{P(0,3)+P(1,3)+P(2,3)+P(3,3)} .
\end{aligned}=
$$

From $\frac{d P(2,1)}{d t}=0$, we have

$$
\begin{aligned}
& 2 P(2,1)=\frac{P(2,3) P(3,1) p+P(0,3) P(3,1) p}{P(0,3)+P(1,3)+P(2,3)+P(3,3)}+\frac{P(2,3) P(3,1) p+P(2,3) P(3,0) p}{P(3,0)+P(3,1)+P(3,2)+P(3,3)} \\
& +\frac{P(2,0) P(0,1)+P(2,0) P(0,3) p}{P(0,0)+P(0,1)+P(0,2)+P(0,3)}+\frac{P(2,0) P(0,1)+P(3,0) P(0,1) p}{P(0,0)+P(1,0)+P(2,0)+P(3,0)} .
\end{aligned}
$$


From $\frac{d P(2,0)}{d t}=0$, we have

$$
\begin{aligned}
& \frac{P(2,0) P(0,1)+P(2,0) P(0,3) p}{P(0,0)+P(0,1)+P(0,2)+P(0,3)}+P(2,0)= \\
& \frac{P(0,3) P(3,0) p+P(2,3) P(3,0) p}{P(0,3)+P(1,3)+P(2,3)+P(3,3)}+\frac{P(2,0) P(0,0)+P(3,0) P(0,0) p}{P(0,0)+P(1,0)+P(2,0)+P(3,0)}+\frac{P(2,2) P(2,0)+P(2,2) P(2,1)}{P(2,0)+P(2,1)+P(2,2)+P(2,3)} .
\end{aligned}
$$

From $\frac{d P(1,3)}{d t}=0$, we have

$$
\begin{aligned}
& \frac{P(1,2) P(2,1)+P(1,2) P(2,3) p}{P(2,0)+P(2,1)+P(2,2)+P(2,3)}+P(3,1) p= \\
& \frac{P(2,1) P(1,3)+P(3,1) P(1,3) p}{P(0,1)+P(1,1)+P(2,1)+P(3,1)}+\frac{P(2,1) P(1,3)+P(0,1) P(1,3)}{P(0,1)+P(1,1)+P(2,1)+P(3,1)}+\frac{P(1,3) P(3,1) p+P(1,3) P(3,0) p}{P(3,0)+P(3,1)+P(3,2)+P(3,3)} .
\end{aligned}
$$

From $\frac{d P(1,2)}{d t}=0$, we have

$$
\begin{aligned}
& \frac{P(2,1) P(1,2)+P(3,1) P(1,2) p}{P(0,1)+P(1,1)+P(2,1)+P(3,1)}+\frac{P(1,2) P(2,1)+P(1,2) P(2,3) p}{P(2,0)+P(2,1)+P(2,2)+P(2,3)}+\frac{P(0,1) P(1,2)+P(2,1) P(1,2)}{P(0,1)+P(1,1)+P(2,1)+P(3,1)}+ \\
& \frac{P(1,2) P(2,0)+P(1,2) P(2,1)}{P(2,0)+P(2,1)+P(2,2)+P(2,3)}=P(3,0) p+P(0,3) p .
\end{aligned}
$$

From $\frac{d P(1,1)}{d t}=0$, we have

$$
\begin{aligned}
& \frac{P(2,1) P(1,1)+P(3,1) P(1,1) p}{P(0,1)+P(1,1)+P(2,1)+P(3,1)}+\frac{P(2,1) P(1,1)+P(0,1) P(1,1)}{P(0,1)+P(1,1)+P(2,1)+P(3,1)} \\
& \frac{P(1,3) P(3,0) p+P(1,3) P(3,1) p}{P(3,0)+P(3,1)+P(3,2)+P(3,3)}+\frac{P(1,0) P(0,1)+P(1,0) P(0,3) p}{P(0,0)+P(0,1)+P(0,2)+P(0,3)} .
\end{aligned}=
$$

From $\frac{d P(1,0)}{d t}=0$, we have

$$
\begin{aligned}
& \frac{P(2,1) P(1,0)+P(3,1) P(1,0) p}{P(0,1)+P(1,1)+P(2,1)+P(3,1)}+\frac{P(0,1) P(1,0)+P(2,1) P(1,0)}{P(0,1)+P(1,1)+P(2,1)+P(3,1)}+\frac{P(1,0) P(0,1)+P(1,0) P(0,3) p}{P(0,0)+P(0,1)+P(0,2)+P(0,3)}= \\
& \frac{P(1,2) P(2,0)+P(1,2) P(2,1)}{P(2,0)+P(2,1)+P(2,2)+P(2,3)}+P(0,1) .
\end{aligned}
$$

From $\frac{d P(0,3)}{d t}=0$, we have

$$
\begin{aligned}
& \frac{P(2,0) P(0,3)+P(3,0) P(0,3) p}{P(0,0)+P(1,0)+P(2,0)+P(3,0)}+\frac{P(0,3) P(3,0) p+P(0,3) P(3,1) p}{P(3,0)+P(3,1)+P(3,2)+P(3,3)}+P(0,3) p= \\
& \frac{P(0,1) P(1,3)+P(2,1) P(1,3)}{P(0,1)+P(1,1)+P(2,1)+P(3,1)}+\frac{P(0,2) P(2,1)+P(0,2) P(2,3) p}{P(2,0)+P(2,1)+P(2,2)+P(2,3)}+P(2,1) .
\end{aligned}
$$

From $\frac{d P(0,2)}{d t}=0$, we have

$$
\begin{aligned}
& \frac{P(2,0) P(0,2)+P(3,0) P(0,2) p}{P(0,0)+P(1,0)+P(2,0)+P(3,0)}+\frac{P(0,2) P(2,1)+P(0,2) P(2,3) p}{P(2,0)+P(2,1)+P(2,2)+P(2,3)}+\frac{P(0,2) P(2,0)+P(0,2) P(2,1)}{P(2,0)+P(2,1)+P(2,2)+P(2,3)}= \\
& \frac{P(0,1) P(1,2)+P(2,1) P(1,2)}{P(0,1)+P(1,1)+P(2,1)+P(3,1)}+P(2,0) .
\end{aligned}
$$

From $\frac{d P(0,1)}{d t}=0$, we have

$$
\begin{aligned}
& \frac{P(2,0) P(0,1)+P(3,0) P(0,1) p}{P(0,0)+P(1,0)+P(2,0)+P(3,0)}+P(0,1)= \\
& \frac{P(0,1) P(1,1)+P(2,1) P(1,1)}{P(0,1)+P(1,1)+P(2,1)+P(3,1)}+\frac{P(0,3) P(3,0) p+P(0,3) P(3,1) p}{P(3,0)+P(3,1)+P(3,2)+P(3,3)}+\frac{P(0,0) P(0,1)+P(0,0) P(0,3) p}{P(0,0)+P(0,1)+P(0,2)+P(0,3)} .
\end{aligned}
$$

From $\frac{d P(0,0)}{d t}=0$, we have

$$
\begin{aligned}
& \frac{P(2,0) P(0,0)+P(3,0) P(0,0) p}{P(0,0)+P(1,0)+P(2,0)+P(3,0)}+\frac{P(0,0) P(0,1)+P(0,0) P(0,3) p}{P(0,0)+P(0,1)+P(0,2)+P(0,3)} \\
& \frac{P(0,1) P(1,0)+P(2,1) P(1,0)}{P(0,+)+P(2,0)+P(0,2) P(2,1)}
\end{aligned}=
$$

\section{ACKNOWLEDGEMENTS}

We acknowledge the support of National Basic Research Program of China (No.2006CB705500), the NSFC under Project Nos. 10532060, 70601026, 10672160, 10872194, the FANEDD, and the SRF for ROCS, SEM. R.J. acknowledges the support of JSPS. Y.-H. Wu acknowledges the support of the Australian Research Council through a Discovery Project Grant.

[1] B. Derrida, Phys.Rep. 301, 65 (1998); R.A. Blythe and M.R. Evans, J. Phys. A, 46, R333 (2007). 
[2] D. Chowdhury, L. Santen, and A. Schadschneider, Phys. Rep. 329, 199 (2000).

[3] J.T. MacDonald, J.H. Gibbs and A.C. Pipkin, Biopolymers 6, 1 (1968).

[4] G.M. Schütz, Europhys.Lett. 48, 623 (1999).

[5] T. Chou, Phys.Rev. Lett. 80, 85 (1998).

[6] R. Lipowsky, S. Klumpp and T. Nieuwenhuizen, Phys. Rev. Lett. 87, 108101 (2001); S. Klumpp and R. Lipowsky, J. Stat. Phys. 113, 233 (2003); P.Greulich et al., Phys. Rev. E 75, 041905 (2007).

[7] A.Nagar, M. Ha, and H.Park, Phys. Rev. E 77, 061118 (2008); J.de Gier and F. H. Essler, Phys.Rev.Lett. 95, 240601 (2005); V.Popkov et al., J.Phys.A 34, L45 (2001).

[8] M. R. Evans et al., Phys. Rev. Lett. 74, 208 (1995); J. Stat. Phys. 80, 69 (1995); E.Pronina and A.B.Kolomeisky, J.Phys.A 40, 2275 (2007).

[9] A. Parmegianni, T. Franosh and E. Frey, Phys. Rev. Lett. 90, 086601 (2003); Phys. Rev. E 70, 046101 (2004); V.Popkov et al., Phys. Rev. E 67, 066117 (2003); M.R. Evans, R. Juhász and L. Santen, Phys. Rev. E 68, 026117 (2003); K.Nishinari et al., Phys.Rev.Lett. 95, 118101 (2005).

[10] P. F. Arndt, T. Heinzel, and V. Rittenberg, J. Stat. Phys. 90, 783 (1998).

[11] M.R. Evans et al., Phys.Rev.Lett. 80, 425 (1998).

[12] N. Rajewsky, T. Sasamoto, and E. R. Speer, Physica A 279, 123 (2000).

[13] B. Schmittmann, K. Hwang, and R. K. P. Zia, Europhys. Lett. 19, 19 (1992); G. Korniss, B. Schmittmann, and R. K. P. Zia, J. Stat. Phys. 86, 721 (1997); D. A. Adams, B. Schmittmann, and R. K. P. Zia, Phys.Rev.E 75, 041123 (2007).

[14] G. Korniss, B. Schmittmann, and R. K. P. Zia, Europhys. Lett. 45, 431 (1999); J. T. Mettetal, B. Schmittmann, and R. K. P. Zia, Europhys. Lett. 58, 653 (2002).

[15] Y.Kafri et al., Phys.Rev.Lett. 89, 035702 (2002).

[16] Y.Kafri et al., Phys.Rev.E 68, 035101(R), (2003).

[17] I.T.Georgiev et al., Phys.Rev.Lett. 94, 115701 (2005); J.Phys.A 39, 3495 (2006).

[18] G.M.Schütz, Bull. Braz. Math. Soc., New Series 37, 523 (2006).

[19] H.W Lee, V Popkov and D Kim, J.Phys.A 30, 8497 (1997); M. E. Fouladvand and H.W. Lee, Phys.Rev.E 60, 6465 (1999).

[20] V.Popkov and G.M.Schütz, J.Stat.Phys. 112, 523 (2003).

[21] S.A. Janowsky and J.L.Lebowitz, J.Stat.Phys. 77, 35 (1994); Phys.Rev.A 45, 618-625 (1992).

[22] G.Schütz, J.Stat.Phys. 71, 471 (1993).

[23] K.Mallick, J. Phys. A 29, 5375 (1996).

[24] G.Tripathy, M.Barma, Phys. Rev. lett. 78, 3039 (1997).

[25] T. Chou and G. Lakatos, Phys. Rev. lett. 93, 198101 (2004).

[26] A.B. Kolomeisky, J. Phys. A 31, 1153 (1998); R. J. Harris and R. B. Stinchcombe, Phys. Rev. E 70, 016108 (2004); M. E. Foulaadvand et al., Phys. Rev. E 75, 011127 (2007); M. Bengrine, J. Phys. A, 32, 2527 (1999).

[27] R. Barlovic et al., Euro.Phys.J.B 5, 793 (1998).

[28] O. J. O'Loan, M. R. Evans and M. E. Cates: Phys. Rev. E 58, 1404 (1998).

[29] K. Nishinari, D. Chowdhury and A. Schadschneider: Phys. Rev. E 67, 036120 (2003).

[30] A.Kunwar et al., J.Phys.Soc.Jpn. 73, 2979 (2004).

[31] V.Popkov and I.Peschel, Phys.Rev.E 64, 026126 (2001).

[32] M. Schreckenberg, A. Schadschneider, K. Nagel, N. Ito, Phys. Rev. E 51, 2939 (1995); A. Schadschneider, M. Schreckenberg, J. Phys. A 26, L679 (1993).

[33] D. Chowdhury and J. S. Wang, Phys. Rev. E 65, 046126 (2002).

[34] G. Szabo, A. Szolnoki, and L. Bodocs, Phys. Rev. A 44, 6375 (1991).

[35] R. Dickman, Phys. Rev. A 34, 4246 (1986).

[36] Note that the "truck" in Ref.[19] is a dynamic defect moving in opposite direction with the "cars" . As a result, the high density region of "cars" moves upstream constantly.

[37] Note that there is no particle beyond the high density region. As a result, the flow rate could be calculated by a loose cluster approximation or by mapping into a zero range process [29]. 